\title{
Differential Distributed Space-Time Coding for Vehicle-to-Vehicle Networks
}

\author{
Sara Al Maeeni*, Vasileios M. Kapinas*†, Sami Muhaidat*, \\ George K. Karagiannidis*†, and Saleh R. Al-Araji* \\ *Electrical and Computer Engineering Department, Khalifa University, Abu Dhabi, United Arab Emirates \\ Email: \{sara.almaeeni, sami.muhaidat, george.karagiannidis, alarajis\}@kustar.ac.ae \\ ${ }^{\dagger}$ Department of Electrical and Computer Engineering, Aristotle University of Thessaloniki, Greece \\ Email: \{kapinas, geokarag\}@ auth.gr
}

\begin{abstract}
Differential distributed space-time coding (DDSTC) has been proved to be suitable for wireless relay networks, since it can provide spatial diversity without the need for channel state information at neither the transmitter nor the receiver side. However, DDSTC suffers from significant error floor in fast-fading channel conditions with high Doppler frequencies due to rapid time variations. For this reason, multiple-symbol differential detection (MSDD) has been proposed in the past, where the detection process involves a larger window size of the received symbols. So far, differential detection for vehicle-tovehicle (V2V) networks has been studied only for single Rayleigh channels. However, experimental and theoretical studies report that double Rayleigh can be considered as an appropriate fading channel model for V2V networks. In this paper, we assess the error performance of a DDSTC scheme operating in a V2V network using MSDD. Simulation results confirm that the error performance of such a system can be improved significantly with MSDD under different channel time-variation scenarios.
\end{abstract}

Index Terms-Differential detection, distributed space-time coding, double Rayleigh, multiple-symbol differential detection, time-varying channel, vehicle-to-vehicle networks.

\section{INTRODUCTION}

Multiple-input multiple-output (MIMO) is an effective technology for improving the spectral efficiency and/or link reliability of wireless communications systems. However, the implementation of multiple antennas at the transmitter and/or receiver side is not practical in some cases, such as in cellular mobile terminals, and in wireless sensor nodes. Cooperative communication has been proposed in the past to exploit the diversity achieved in a distributed manner by using singleantenna nodes. It consists of three primary types of elements, the source node $S$, one or more relay nodes $R$, and the destination node $D$. The source is assisted by at least one relay in order to transmit the signal to the destination. Various cooperative relaying protocols have been proposed in the literature so far, the most popular being the decode-andforward (DF), and the amplify-and-forward (AF) protocols [1]. In AF, the relay simply amplifies the signal by a pre-specified amplification factor and transmits it to the destination node. Due to its many advantages, the AF relaying strategy has been included in the long term evolution (LTE)-advanced physical layer specifications [2], [3].

Vehicular ad hoc network (VANET) is a promising intelligent transportation system technology, which enables vehicles to communicate with each other and roadside stations. It can support critical vehicular safety applications, such as emergency warning, collision avoidance, road condition broadcasting, and lane-changing assistance [4]-[6]. Due to their advantages in terms of road safety, efficiency, and comfort, VANETs have received a lot of attention in the last couple of years. Therefore, many efforts have been made towards the design of robust architectures for vehicle-to-vehicle (V2V) and vehicle-to-road (V2R) networks, such as the cooperative communication scheme proposed in [7] for enhancing the coverage and link reliability in vehicular networks.

Most of existing works in the literature assume perfect channel state information (CSI) at $D$. However, in practical scenarios, the CSI is unknown and it has to be obtained with the aid of pilot symbols. Besides, allocating specific resources for these symbols, the correct estimation of channel gains would be very difficult or even infeasible in fast-fading channels, where the channel gains vary rapidly. It comes out that, the design of a decoder that does not require CSI is of major importance in $\mathrm{V} 2 \mathrm{~V}$ systems.

Differential detection based on two-symbol differential detection (TSDD) has been proved to be a good practical solution in the absence of CSI. It has been shown that TSDD performs $3-4 \mathrm{~dB}$ worse than the coherent detection. However, in high speed scenarios, the assumption that the consecutive channel gains are constant does not hold any more. Thus, the TSDD suffers from high error floor and severe bit error rate (BER) degradation. Recently, a generalized likelihood sequence detector (GLSD) using the destination noise variance has been proposed [8]. This detector outperforms the diversity combiner technique and achieves near maximum-likelihood detection (MLD) performance with the same complexity. Multiple symbol differential detection (MSDD) has been proposed in [9]. This type of detection processes a larger window size of the received symbols (with respect to the TSDD), thus significantly improving the BER performance. Concerning MIMO systems, a low complexity MSDD for unitary spacetime codes based on sphere decoding has been proposed in [10]. However, in all cases, the computational complexity of MSDD increases exponentially with the window size.

Although differential detection for $\mathrm{V} 2 \mathrm{~V}$ networks has been studied extensively in the literature, the channel model con- 


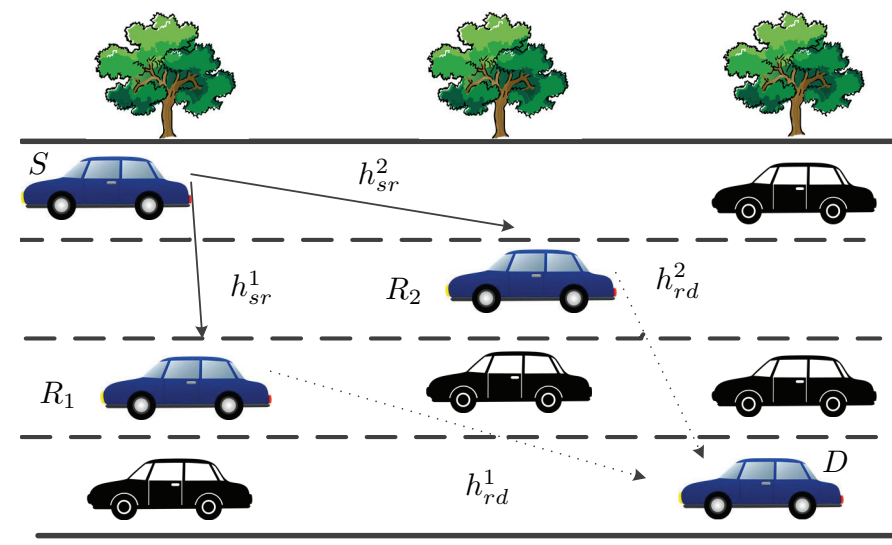

Fig. 1. Cooperative wireless relay system.

sidered in all cases is (single) Rayleigh [11], [12]. However, experimental and theoretical studies report that double (or cascaded) Rayleigh can be considered as an appropriate fading channel model for V2V systems [13], [14]. This paper investigates the performance of a differential space-time coding scheme for $\mathrm{V} 2 \mathrm{~V}$ systems with AF relaying employing a low-complexity MSDD under three different channel time variations, namely low, medium, and fast. The outline of the paper is as follows; Section II presents the system model. Section III deals with the TSDD and the MSDD and their performance over fast fading channel conditions. Finally, the simulation results are presented in Section IV for both the single and double Rayleigh channels.

Notations: Bold upper-case and lower-case letters denote matrices and vectors, respectively. Also, $(.)^{*}$, and $(.)^{H}$ stand for the complex conjugate, and the Hermitian transpose of a complex vector or matrix, respectively. Finally, $\mathcal{C N}$ is the complex Gaussian distribution.

\section{SySTEM MODEL}

Consider a cooperative half-duplex V2V scheme consisting of one source node $S$, two relay nodes $R_{1}, R_{2}$, and one destination node $D$ in a highway/suburban area, as shown in Fig. 1. Each terminal in the network is equipped with a single antenna. The relaying scheme implements the Alamouti space-time code in a distributive manner with the aid of the AF protocol. Two phases are assumed in the transmission process; in Phase I, $S$ transmits to $R_{1}$ and $R_{2}$, while in Phase II the two relays forward the amplified received signals to $D$. All links are considered to be affected by the product of two independent Rayleigh channel gains. The envelope of this cascaded Rayleigh channel model has probability density function given by [15]

$$
P_{z}[z]=2 z K_{0}(z \sqrt{2}),
$$

where $K_{0}$ is the zero-order modified Bessel function of the second kind and $z$ is a random variable. Moreover, the secondorder statistics follows Jakes' model, which is given by [15]

$$
R(\tau)=J_{0}\left(2 \pi f_{1} \tau\right) J_{0}\left(2 \pi f_{2} \tau\right),
$$

where $J_{0}$ is the zero-order Bessel function of the first kind. Additionally, all channels between individual nodes are perturbed by independent samples of additive white Gaussian noise (AWGN). Moreover, the information bits to be broadcasted by $S$ are converted to symbols using phase shift keying (PSK) modulation. According to [16], for each codeword, one out of the $L$ entries of the set of $2 \times 2$ unitary matrices given by $\mathcal{S}=\left\{\mathbf{S}_{l} \mid \mathbf{S}_{l}^{H} \mathbf{S}_{l}=\mathbf{S}_{l} \mathbf{S}_{l}^{H}=\mathbf{I}_{2}\right\}$ is selected, where $l=1, \ldots, L$ and $L$ is the total number of codewords.

The main purpose of differential modulation is to make the receiver structure simple by omitting the need for channel estimation and phase tracking. Therefore, the differential encoding is based on modulating the information through the phase difference between two consecutive symbols. Hence, at time instant $n$, the modulated information symbols $\mathbf{S}[n] \in \mathcal{S}$ at the source are differentially encoded according to

$$
\mathbf{x}[n]=\mathbf{S}[n] \mathbf{x}[n-1], \quad n=1,2, \ldots, N,
$$

where $\mathbf{x}[n]$ denotes the transmitted signal with $\mathbf{x}[0]=\left[\begin{array}{ll}1 & 0\end{array}\right]^{t}$ being the initial reference symbol, $N$ is the number of data symbols within one frame, and

$$
\mathbf{S}[n]=\frac{1}{\sqrt{2}}\left[\begin{array}{rr}
s_{1}(n) & -s_{2}^{*}(n) \\
s_{2}(n) & s_{1}^{*}(n)
\end{array}\right] .
$$

During Phase I, $S$ broadcasts the vector $\mathbf{x}[n]$ over the relay channels. The received vector by the $i$ th relay, $i=1,2$, is

$$
\mathbf{y}_{s r}^{i}[n]=\sqrt{P_{s}} h_{s r}^{i}[n] \mathbf{x}[n]+\mathbf{w}_{r}^{i}[n],
$$

where $P_{s}$ denotes the transmission power at $S, h_{s r}^{i}$ is the $S \rightarrow R_{i}$ channel assumed to be double Rayleigh distributed with $\sqrt{h_{s r}^{i}} \sim \mathcal{C N}(0,1)$, and $\mathbf{w}_{r}^{i}[n] \sim \mathcal{C N}\left(\mathbf{0}, N_{0} \mathbf{I}_{2}\right)$ is the AWGN sample at $i$ th relay.

During Phase II, the relays form the distributed Alamouti space-time code by combining the following matrices [16]

$$
\mathbf{v}_{s r}^{i}[n]=\mathbf{A}^{i} \mathbf{y}_{s r}^{i}[n]+\mathbf{B}^{i} \mathbf{y}_{s r}^{i *}[n],
$$

where $\mathbf{A}^{i}, \mathbf{B}^{i}$ for $i=1$ and $i=2$ are defined respectively as

$$
\mathbf{A}^{1}=\left[\begin{array}{ll}
1 & 0 \\
0 & 1
\end{array}\right], \mathbf{B}^{1}=0, \mathbf{A}^{2}=0, \mathbf{B}^{2}=\left[\begin{array}{rr}
0 & -1 \\
1 & 0
\end{array}\right] .
$$

Since the system does not have any CSI, and in order to ensure the minimum pairwise-error probability (PEP), each relay amplifies the received signal by a fixed amplification factor $\alpha_{r}=\sqrt{P_{r}^{i} /\left(P_{s}+N_{0}\right)}$, where $P_{r}^{i}$ is the average power per symbol at $R_{i}$. Thus, the total signal received at $D$ becomes

$$
\mathbf{y}[n]=\sum_{i=1}^{2} \alpha_{r} h_{r d}^{i}[n] \mathbf{v}_{s r}^{i}[n]+\mathbf{w}_{d}[n]
$$

where $h_{r d}^{i}$ is the $R_{i} \rightarrow D$ channel assumed to be double Rayleigh distributed with $\sqrt{h_{r d}^{i}} \sim \mathcal{C N}(0,1)$, and $\mathbf{w}_{d}[n] \sim$ $\mathcal{C N}\left(\mathbf{0}, N_{0} \mathbf{I}_{2}\right)$ is the AWGN sample at $D$. 


\section{DiffEREnTIAL Detection}

\section{A. Two-Symbol Differential Detection}

The channel is assumed to be fixed for two consecutive symbols, i.e., $\mathbf{h}[n] \approx \mathbf{h}[n-1]$. Thus, the received vector reads

$$
\mathbf{y}[n]=\mathbf{S}[n] \mathbf{y}[n-1]+\mathbf{w}_{d}[n]-\mathbf{S}[n] \mathbf{w}_{d}[n-1]
$$

Then, the decoding rule is given as

$$
\widehat{\mathbf{S}}[n]=\arg \min _{\mathbf{S}[n] \in \mathcal{S}}\|\mathbf{y}[n]-\mathbf{S}[n] \mathbf{y}[n-1]\| .
$$

However, in fast fading, the two consecutive symbols are no longer fixed and the change between them becomes non-trivial, let say equal to $\Delta \mathbf{h}$. In this case, (9) can be expressed as

$$
\mathbf{y}[n]=\mathbf{S}[n] \mathbf{y}[n-1]+\widetilde{\mathbf{w}}_{d}[n],
$$

where

$$
\widetilde{\mathbf{w}}_{d}[n]=\mathbf{w}_{d}[n]-\mathbf{S}[n] \mathbf{w}_{d}[n-1]+a_{r} \sqrt{P_{s}} \mathbf{x}[n] \Delta \mathbf{h} .
$$

Obviously, the increase in noise power depends on both $\sqrt{P_{s}}$ and $\Delta \mathbf{h}$. Thus, the conventional differential detection experiences severe error degradation in a fast-fading channel.

\section{B. Multiple-Symbol Differential Detection}

The basic idea of MSDD is based on increasing the observation window of the received symbols. The technique makes use of maximum-likelihood sequence estimation (MLSE) of the transmitted phases rather than symbol-by-symbol maximumlikelihood (ML) detection as in TSDD [9], [11]. As such, the performance of this multiple-symbol detection scheme fills the gap between TSDD of PSK and ideal coherent of PSK with differential encoding. The amount of improvement gained over the conventional differential detection depends on the number of additional symbol intervals added to the observation. What is particularly interesting is that substantial performance improvement can be obtained for only one or two additional symbol intervals of observation [9].

Simply put, an MSDD is a decoder that makes a decision about a block of $K$ consecutive PSK symbols based on $K+1$ received samples. The first received sample is used to provide a phase reference for the entire block while the last sample is used to provide a reference for the next block. Obviously, for $K=2$, MSDD reduces to the TSDD. The larger the value of $K$, the better the error performance. In the limiting case where $K$ approaches infinity, the performance of a MSDD in an AWGN channel approaches that of a coherent detector with differential encoding to resolve phase ambiguity [17].

The ML-based MSDD has better BER performance than many other detectors intended for differential modulation. However, the computational complexity of ML-based MSDD quickly becomes prohibitive as the observation window size $K$ grows. While low-complexity MSDD algorithms for the time-invariant Rayleigh fading channel have been considered before, there is a need for low-complexity MSDD algorithms for general time-varying Rayleigh fading channels. Towards this end, a polynomial-time complexity approach, called semidefinite relaxation, has been suggested in [18] in order to achieve differential detection with near-ML performance.
TABLE I

SEVERIty ScEnARIOS USED In Simulations $(i=1,2)$

\begin{tabular}{|c|c|c|c|}
\hline case ID & channel-type & $f_{d}$ for $S-R$ & $f_{d}$ for $R-D$ \\
\hline$\# 1$ & low-fading & 0.0001 & 0.0001 \\
\hline$\# 2$ & medium-fading & 0.005 & 0.003 \\
\hline$\# 3$ & fast-fading & 0.01 & 0.009 \\
\hline
\end{tabular}

\section{Simulation Results}

Monte Carlo simulations have been conducted in order to evaluate the BER performance of the differential distributed space-time coding (DDSTC) scheme, which has been considered to operate over double Rayleigh flat fading for all $S \rightarrow R_{1}, R_{2}$ and $R_{1}, R_{2} \rightarrow D$ links. Different cases represent different normalized Doppler frequency $f_{d}$ values from sourceto-relay $\left(S-R_{i}\right)$ and relay-to-destination $\left(R_{i}-D\right)$, where $i=$ 1,2 . In this simulation, the Doppler frequency is a function of the symbol period, the vehicle speed, and the carrier frequency. For the low channel severity case, it is assumed that the system operates in slow fading, which corresponds to small values of the normalized Doppler frequency, namely $f_{d}=0.0001$ for all links. The medium channel severity case assumes medium fading, such that the normalized Doppler frequencies are 0.005 and 0.003 for $S-R_{i}$ and $R_{i}-D$, respectively. High channel severity conditions are also assumed, where the normalized Doppler frequencies are 0.01 and 0.009 for $S-R_{i}$ and $R_{i}-D$, respectively, as shown in Table I. Notice that, the power is allocated as follows; $P_{s}=0.5, P_{r}^{1}=0.25$, and $P_{r}^{2}=0.25$.

In all simulations, the information bits are first modulated using either binary PSK (BPSK) or Quadrature PSK (QPSK). Then, the modulated symbols are differentially encoded and transmitted through the two phases described in Sec II. Both single Rayleigh and double Rayleigh fading channel conditions are considered in each link. The evaluated systems are shown in Figs. 2, 3 and 4 for different cases. As shown in Fig. 2, the BER performance over single Rayleigh with QPSK for Case $I$ is decreased with the signal-to-noise ratio (SNR). However, the error floor starts to appear in Case II after 30 $\mathrm{dB}$, while for Case III the error floor starts earlier at $25 \mathrm{~dB}$. This is due to the fact that the assumption of the phase being constant over several symbol intervals is not valid. Therefore, the rapid change in phase is very difficult to be tracked by TSDD. Interestingly, the increase in the observation window size of the received symbols up to $K=10$ can eliminate this degradation in Cases II and III, as shown in Figs. 3 and 4.

Given the improved error performance in single Rayleigh channel using MSDD, the error performance of double Rayleigh with BPSK is assessed in Fig. 3. As expected, the BER performance over double Rayleigh degrades severely compared to the single Rayleigh channel. The TSDD leads to an error floor at $1.5 \times 10^{-3}$ for Case II. Also, it can be noticed that, using TSDD leads to an error floor at $1.8 \times 10^{-2}$ for Case III. However, the BER using MSDD can be significantly improved for both medium and high time-varying fading.

The BER of TSDD and MSDD with QPSK is shown in Fig. 4. In Case II, the BER starts to flat out after 35 


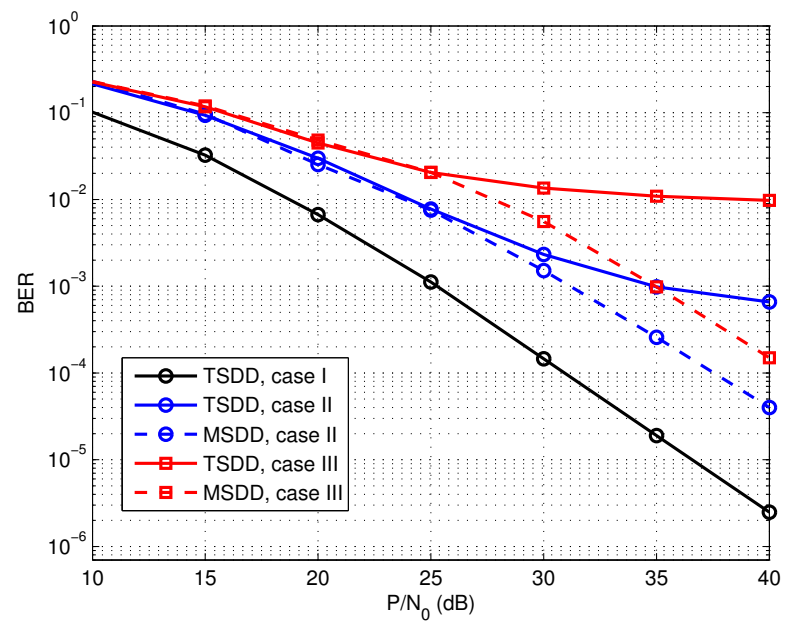

Fig. 2. BER for single Rayleigh and QPSK in different channel variations.

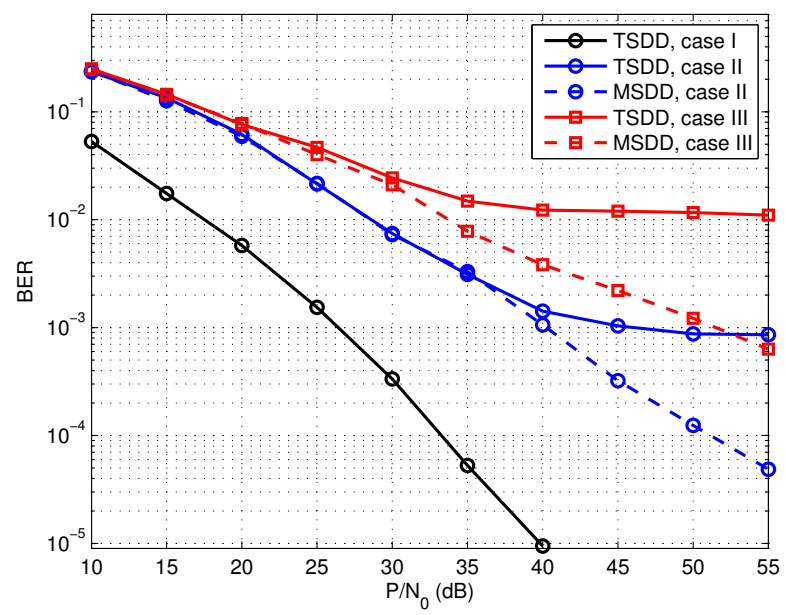

Fig. 3. BER for double Rayleigh and BPSK in different channel variations.

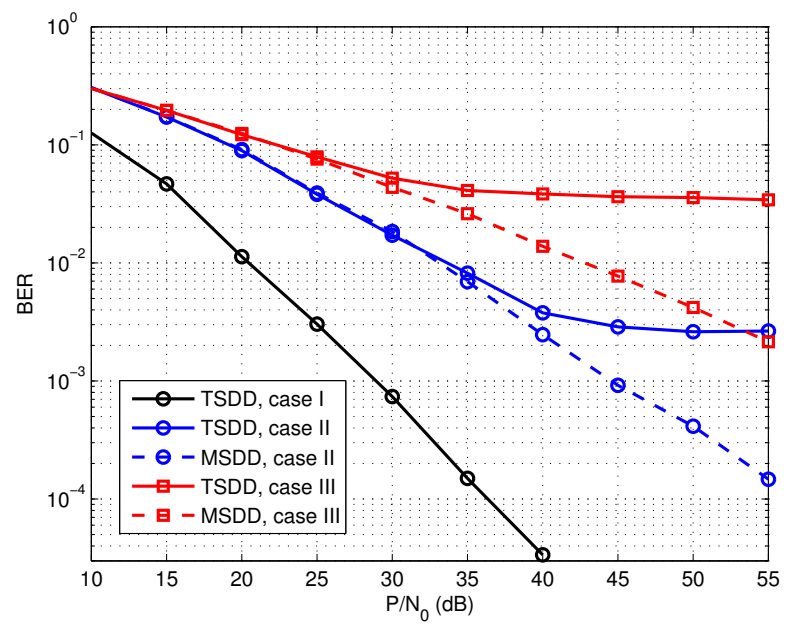

Fig. 4. BER for double Rayleigh and QPSK in different channel variations.
$\mathrm{dB}$ due to the channel gain difference. As this difference increases in Case III, the error floor starts to appear around $25 \mathrm{~dB}$. Applying MSDD in this channel improves the BER significantly and provides a coding gain advantage of $5 \mathrm{~dB}$ at a BER of $3 \times 10^{-3}$ for Case II and much more for Case III.

Simulation results confirm that the BER performance of DDSTC in practical V2V networks can benefit from the use of MSDD for all levels of time-variation (channel severity).

\section{REFERENCES}

[1] P. Liu, S. Gazor, I.-M. Kim, and D. I. Kim, "Noncoherent amplifyand-forward cooperative networks: Robust detection and performance analysis," IEEE Trans. Commun., vol. 61, no. 9, pp. 3644-3659, Sep. 2013.

[2] C. Xing, S. Ma, Z. Fei, Y.-C. Wu, and H. V. Poor, "A general robust linear transceiver design for multi-hop amplify-and-forward MIMO relaying systems," IEEE Trans. Signal Processing, vol. 61, no. 5, pp. 1196-1209, Mar. 2013.

[3] E. Dahlman, S. Parkvall, and J. Skold, 4G: LTE/LTE-Advanced for Mobile Broadband, 1st ed. Academic Press, 2011.

[4] H. Wang, R. P. Liu, W. Ni, W. Chen, and I. B. Collings, "VANET modeling and clustering design under practical traffic, channel and mobility conditions," IEEE Trans. Commun., vol. 63, no. 3, pp. 870-881, Mar. 2015.

[5] G. Karagiannis, O. Altintas, E. Ekici, G. Heijenk, B. Jarupan, K. Lin, and T. Weil, "Vehicular networking: A survey and tutorial on requirements, architectures, challenges, standards and solutions," IEEE Commun. Surveys Tuts, vol. 13, no. 4, pp. 584-616, Fourth 2011.

[6] S. Zeadally, R. Hunt, Y.-S. Chen, A. Irwin, and A. Hassan, "Vehicular ad hoc networks (vanets): Status, results, andchallenges," Telecommun. Syst., vol. 50, no. 4, pp. 217-241, 2012.

[7] M. F. Feteiha and M. Uysal, "Cooperative transmission for broadband vehicular networks over doubly selective fading channels," IET Commun., vol. 6, no. 16, pp. 2760-2768, Nov. 2012.

[8] P. Liu, S. Gazor, and I. Kim, "A practical differential receiver for amplify-and-forward relaying," IEEE Wireless Commun. Lett., vol. 3 , no. 4 , pp. 349-352, Aug. 2014

[9] D. Divsalar and M. K. Simon, "Multiple-symbol differential detection of MPSK," IEEE Trans. Commun., vol. 38, no. 3, pp. 300-308, Mar. 1990.

[10] V. Pauli and L. Lampe, "Tree-search multiple-symbol differential decoding for unitary space-time modulation," IEEE Trans. Commun., vol. 55, no. 8, pp. 1567-1576, Aug. 2007.

[11] M. R. Avendi, H. H. Nguyen, and N. Quoc-Tuan, "Multiple-symbol differential detection for distributed space-time coding," in Proc. International Conference on Computing, Management and Telecommunications, Apr. 2014, pp. 62-66.

[12] M. R. Avendi and H. H. Nguyen, "Differential dual-hop relaying under user mobility," IET Commun., vol. 8, no. 17, pp. 3161-3169, 2014.

[13] R. U. Nabar, H. Bolcskei, and F. W. Kneubuhler, "Fading relay channels: performance limits and space-time signal design," IEEE J. Select. Areas Commun., vol. 22, no. 6, pp. 1099-1109, Aug. 2004.

[14] V. Erceg, S. J. Fortune, J. Ling, A. J. Rustako, and R. A. Valenzuela, "Comparisons of a computer-based propagation prediction tool with experimental data collected in urban microcellular environments," IEEE J. Select. Areas Commun., vol. 15, no. 4, pp. 677-684, May 1997.

[15] Y. Ibdah, Y. Ding, H. M. Kwon, and K. Lee, "Simulations on the statistical properties for cascaded Rayleigh fading channel," in Proc. Military Communications Conference MILCOM, Nov. 2011, pp. 435440.

[16] Y. Jing and H. Jafarkhani, "Distributed differential space-time coding for wireless relay networks," IEEE Trans. Commun., vol. 56, no. 7, pp. 1092-1100, Jul. 2008.

[17] P. Ho and D. Fung, "Error performance of multiple-symbol differential detection of PSK signals transmitted over correlated Rayleigh fading channels," IEEE Trans. Commun., vol. 40, no. 10, pp. 1566-1569, Oct. 1992.

[18] Z. Ma, P. Fan, E. G. Larsson, and B. Honary, "Quasi-maximumlikelihood multiple-symbol differential detection for time-varying Rayleigh fading channel," Electron. Lett., vol. 45, no. 22, pp. 11271128, Oct. 2009. 